\title{
Quantum-information processing using Josephson junctions coupled through cavities
}

\author{
Shi-Liang Zhu, ${ }^{1,2}$ Z. D. Wang, ${ }^{1,3, *}$ and Kaiyu Yang ${ }^{1}$ \\ ${ }^{1}$ Department of Physics, University of Hong Kong, Pokfulam Road, Hong Kong, China \\ ${ }^{2}$ Department of Physics, South China Normal University, Guangzhou, China \\ ${ }^{3}$ Department of Material Science and Engineering, University of Science and Technology of China, Hefei, China
}

(Received 24 April 2003; published 10 September 2003)

\begin{abstract}
Josephson junctions have been shown to be a promising solid-state system for implementation of quantum computation. The two-qubit gates are generally realized by the capacitive coupling between the nearestneighbor qubits. We propose an effective Hamiltonian to describe charge qubits coupled through the microwave cavity. We find that nontrivial two-qubit gates may be achieved by this coupling. The ability to interconvert localized charge qubits and flying qubits in the proposed scheme implies that quantum network can be constructed using this large scalable solid-state system.
\end{abstract}

DOI: 10.1103/PhysRevA.68.034303

PACS number(s): 03.67.Lx, 03.67.Hk, 85.25.Cp

Quantum-information processing (QIP) with a large number of qubits is now attracting increasing interest. So far, a number of systems have been proposed as potentially viable qubit models. Among a variety of qubits implemented, solidstate qubits are of particular interest because of their potential suitability for integrated devices. Charge qubits based on Josephson junctions have been shown to be a promising solid-state candidate for implementation of quantum computation (QC) [1-8]. QIP tasks usually involve not only computation but also communication. However, whether Josephson junctions are also suitable for quantum communication is still an important open question.

The basic criteria for QIP have been described in Refs. $[9,10]$. Among them, realization of a universal set of quantum gates plays a central role in QC. Besides that the gates can act on any pair of qubits is also a necessary element for fault tolerant computation [10]. Moreover, the ability to interconvert stationary and flying qubits, and to faithfully transmit flying qubits between specified nodes are also required for quantum communication [9].

In this paper, we show that a new system consisting of Josephson junctions coupled through microwave cavities fulfills the above requirements, and thus is a promising candidate for QIP. This system possesses at least three distinctive merits.

(i) A serious limitation of solid-state computers is that the decoherence time in these systems is relatively short. However, from the report in a recent experiment, it is possible that quantum coherence of a large number qubits may be easier to maintain if junctions locate within a high quality microwave cavity [11].

(ii) The nontrivial two-qubit gate acting on any pair of qubits can be realized, and possible fault tolerant geometric quantum computation [5-7] proposed in the absent of cavity is still workable. Thus the combination of different fault tolerant approaches is possible and may be helpful for overcoming the infamous decoherence effects. Here we provide a new experimentally feasible method to realize two-qubit gates: coupling charge qubits through a high quality cavity,

*Electronic address: zwang@hkucc.hku.hk and show that the combination of capacitive as well as cavity coupling in symmetric superconducting quantum interference device (SQUID) or just the cavity coupling in asymmetric SQUID allow the implementation of two-qubit gates between any pair of qubits.

(iii) The present scheme is able to interconvert stationary charge qubits and flying photon qubits, and faithfully transmit flying qubits between specified nodes in quantum network. The ideal quantum transmission between charge qubits in different cavities can be achieved by cavity QED techniques [12]. Thus the quantum network based on solid-state quantum computers may be connected by using transmission fibre, and photons as flying qubits in the scheme clearly represent the best qubit carrier for fast and reliable communication over long distances.

The single-Josephson-junction qubit we considered is shown in Fig. 1(a) [3]. It consists of a small superconducting box with $n$ excess Cooper-pair charges, formed by a SQUID with capacitances $C_{J m}(m=1,2)$ and Josephson coupling energies $E_{J m}$, pieced by a magnetic flux $\phi$. A control gate voltage $V_{g}$ is connected to the system via a gate capacitor $C_{g}$. The Hamiltonian of the system becomes

$$
H=E_{c h}(n-\bar{n})^{2}-E_{J 1} \cos \gamma_{1}-E_{J 2} \cos \gamma_{2},
$$

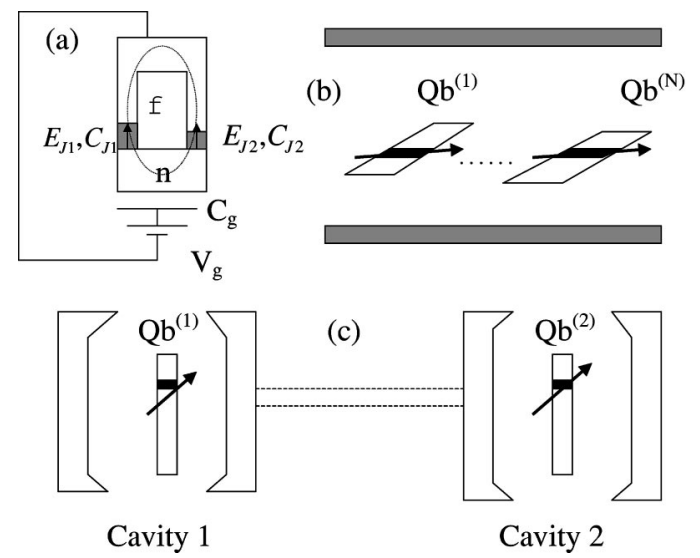

FIG. 1. Josephson qubit systems. (a) A single-Josephson qubit. (b) Josephson qubits in a cavity. (c) Josephson qubits in cavities connected by transmission fibre. 
where $n$ is the number operator of (excess) Cooper-pair charges on the box, $E_{c h}=2 e^{2} /\left(C_{g}+C_{J 1}+C_{J 2}\right)$ is the charging energy, $\bar{n}=C_{g} V_{g} / 2$ is the induced charge and can be controlled by changing $V_{g} \cdot \gamma_{m}$ is the gauge-invariant phase difference between points on opposite sides of the $m$ th junction. Assuming that the Josephson junction locates within a single-mode resonant cavity, then $\gamma_{m}=\varphi_{m}$ $-\left(2 \pi / \phi_{0}\right) \int_{l_{m}} \mathbf{A}_{m} \cdot d \mathbf{l}_{m}$, where $\varphi_{m}$ is the phase difference (of the superconducting wave function) across the $m$ th junction in a particular gauge, and may take the same value $\varphi$ [13]. $\mathbf{A}_{m}$ is the vector potential in the same gauge, and the line integral is taken across the $m$ th junction and along the arrows in Fig. 1(a). $\mathbf{A}_{m}$ may be divided into two parts $\mathbf{A}_{m}^{\prime}+\mathbf{A}_{m}^{\phi}$, where the first term arises from the electromagnetic field of the cavity normal mode (which can be described as an oscillator) and the second term arises from the magnetic flux $\phi$. In the Coulomb gauge, $\mathbf{A}_{m}^{\prime}$ takes the form $\sqrt{\hbar / 2 \omega V}(a$ $\left.+a^{\dagger}\right) \hat{\epsilon}[13]$, where $\hat{\epsilon}$ is the unit polarization vector of the cavity mode, $V$ is the volume of the cavity, $a$ and $a^{\dagger}$ are the annihilation and creation operators for the quantum oscillators, and $\omega$ is its frequency. Therefore, we have

$$
\frac{2 \pi}{\phi_{0}} \int_{l_{m}} \mathbf{A}_{m} \cdot d \mathbf{l}_{m}=\frac{2 \pi}{\phi_{0}} \int_{l_{m}} \mathbf{A}_{m}^{\phi} \cdot d \mathbf{l}_{m}+g\left(a+a^{\dagger}\right),
$$

where $g=2 e \hat{\epsilon} \cdot \mathbf{l} / \sqrt{2 \varepsilon \omega V \hbar}$ is the coupling constant between the junctions and the cavity, with $l$ the thickness of the insulating layer in the junction. For simplicity, we assume that $g_{m}=g$. As for $\mathbf{A}^{\phi}$, we have another constraint: $\oint_{C} \mathbf{A}^{\phi} d \mathbf{l}$ $=\phi$, where the integral path $C$ is along the dashed line in Fig. 1(a).

We consider systems in the charging regime where $E_{c h}$ $\gg E_{J m}$, then a convenient basis is formed by the charge states, parametrized by the number of Cooper pairs $n$ on the box, and $\varphi$ is its conjugate, $n=-i \hbar \partial / \partial(\varphi)$. They satisfy the standard commutation relation: $[\varphi, n]=i$. In this basis Hamiltonian (1) reads

$$
\begin{aligned}
H= & \sum_{n}\left[E_{c h}(n-\bar{n})^{2}|n\rangle\langle n|-\frac{E_{J}(\phi)}{2}\left(e^{-i\left[g\left(a+a^{\dagger}\right)+\beta\right]}|n+1\rangle\right.\right. \\
& \times\langle n|+\text { H.c. })],
\end{aligned}
$$

where

$$
\begin{gathered}
\tan \beta=\frac{E_{J 1}-E_{J 2}}{E_{J 1}+E_{J 2}} \tan \left(\frac{\pi \phi}{\phi_{0}}\right), \\
E_{J}(\phi)=\sqrt{\left(E_{J 1}-E_{J 2}\right)^{2}+4 E_{J 1} E_{J 2} \cos ^{2}\left(\pi \phi / \phi_{0}\right)},
\end{gathered}
$$

with $\phi_{0}=\pi \hbar / e$ being the flux quantum. At temperature much lower than the charging energy and the gate voltage tuning close to a degeneracy $(\bar{n} \sim 1 / 2)$, the relevant physics is captured by considering only the two charge eigenstates $n=0,1$, which constitute the basis $\{|0\rangle,|1\rangle\}$ of the computation Hilbert space of the qubit.
If we have $N$ such qubits located within a single-mode cavity [Fig. 1(b)], to a good approximation, the total system can be considered as $N$ two-state systems coupled to a quantum harmonic oscillator [13]. In this case, the system considered here can be described by the Hamiltonian $H=H_{0}$ $+H_{\text {int }}$, where

$$
\begin{gathered}
H_{0}=\hbar \nu\left(a^{\dagger} a+\frac{1}{2}\right)+\sum_{j}^{N} E_{n_{k}}^{-} \sigma_{j}^{z}, \\
H_{i n t}=-\frac{1}{2} \sum_{j}^{N} E_{J}\left(\phi_{j}\right)\left(e^{-i\left[g\left(a+a^{\dagger}\right)+\beta_{j}\right]} \sigma_{j}^{+}+\text {H.c. }\right),
\end{gathered}
$$

with $E_{\bar{n}_{k}}=E_{c h}\left(\bar{n}_{k}-1 / 2\right)$. A spin notation is used for the qubit $j$ with Pauli matrices $\left\{\sigma_{j}^{x}, \sigma_{j}^{y}, \sigma_{j}^{z}\right\}$, and $\sigma_{j}^{ \pm}=\left(\sigma_{j}^{x}\right.$ $\left.\pm i \sigma_{j}^{y}\right) / 2$. For simplicity, we have assumed the same $E_{c h}$, $E_{J 1}$, and $E_{J 2}$ for all different qubits. The tunable parameters $E_{J}\left(\phi_{j}\right)$ and $\beta_{j}$ have the same forms as those in Eqs. (4) and (5), where $\phi_{j}$ is the magnetic flux pieced the $j$ th Josephson charge qubit. It is remarkable that the main parameters $E_{\bar{n}_{k}}$ and $E_{J}\left(\phi_{j}\right)$ in the Hamiltonian can be controlled independently for every qubit. Furthermore, Eq. (7) representing the interaction between charge qubits and cavity QED is essential in the implementation of QIP.

We now present two examples to demonstrate that QC may be accomplished by using the above Josephson-junction system. For universal QC, we need to realize only two kinds of noncommutable single-qubit gates and one nontrivial twoqubit gate [14].

The first example is QC using asymmetric SQUID loop $\left(E_{J 1} \neq E_{J 2}\right)$. The single-qubit gates can be realized when a qubit energy gap is far from the cavity energy, thus the qubit is decoupled from the cavity. In this case the effective Hamiltonian for qubit $k$ reads [5]

$$
H_{k}=E_{n_{k}} \sigma_{k}^{z}-E_{J}\left(\phi_{k}\right)\left(\sigma_{k}^{x} \cos \beta_{k}-\sigma_{k}^{y} \sin \beta_{k}\right) .
$$

When both $\bar{n}_{k}$ and $\phi_{k}$ are time independent, the evolution operator is obtained explicitly

$$
U\left(\gamma_{k}\right)=\exp \left(-\frac{i}{\hbar} \int_{0}^{t} H_{k} d t\right)=\exp \left(-i \gamma_{k} \sigma_{\mathbf{n}} \cdot \mathbf{n}\right),
$$

where $\mathbf{n}=\left(-E_{J}\left(\phi_{k}\right) \cos \beta_{k}, E_{J}\left(\phi_{k}\right) \sin \beta_{k}, E_{n_{k}}^{-}\right) / E_{k}$, with $E_{k}$ $=\sqrt{E_{J}^{2}\left(\phi_{k}\right)+E_{n_{k}}^{2}}, \gamma_{k}=E_{k} t / \hbar$, and $\sigma_{\mathbf{n}}$ is Pauli matrix along the direction $\mathbf{n}$. We may check that $U\left(\mathbf{n}_{1}\right)$ and $U\left(\mathbf{n}_{2}\right)$ are noncommutable if $\mathbf{n}_{1} \neq \pm \mathbf{n}_{2}$. Consequently, the universal single-qubit gates can be realized by suitably choosing $\bar{n}_{k}$ and $\phi_{k}$.

We now address that nontrivial two-qubit gate may be achieved by the cavity coupling. In the condition that $g \sqrt{n+1}$ is well below unity (the Lamb-Dicke limit), we may expand Eq. (7) in powers of $g$ and neglecting rapidly rotating terms. By choosing the first blue sideband frequency $\left(E_{\bar{n}_{k}}\right.$ $=\hbar \nu)$, we find a transformation 


$$
R_{k}^{+}\left(\theta_{k}, \beta_{k}\right)=\exp \left[-i \frac{\theta_{k}}{2}\left(i e^{-i \beta_{k}} \sigma_{k}^{+} a+\text { H.c. }\right)\right],
$$

with $\theta_{k}=E_{J}\left(\phi_{k}\right) g t / \hbar$. Similarly, by choosing the first red sideband frequency $\left(E_{n_{k}}=-\hbar \nu\right)$, we have another transformation

$$
R_{k}^{-}\left(\theta_{k}, \beta_{k}\right)=\exp \left[-i \frac{\theta_{k}}{2}\left(i e^{-i \beta_{k}} \sigma_{k}^{+} a^{\dagger}+\text { H.c. }\right)\right]
$$

A controlled-NOT gate for control qubit $j$ and target qubit $k$ can be realized by using $R_{k}^{ \pm}$and single-qubit rotations, for example,

$$
\begin{gathered}
U_{j k}^{C N O T}=Z_{j}[-\pi /(2 \sqrt{2})] R_{j}^{-}\left(\pi, \beta_{j}\right) H_{k} P_{k} \\
Z_{k}[-\pi /(2 \sqrt{2})] H_{k} R_{j}^{-}\left(\pi, \beta_{j}\right)
\end{gathered}
$$

for any value of $\beta_{j}$ [15]. Here $Z_{j}(\zeta)$ is a phase gate for qubit $j, H_{k}$ is the Hadamard gate, and $P_{k}$ $=R_{k}^{+}(-\pi / 2,0) R_{k}^{+}(-\pi \sqrt{2},-\pi / 2) R_{k}^{+}(\pi / 2,0)$. The gates described by Eqs. (9) and (10) consist of a universal set of quantum gates using charge qubits in asymmetric SQUID loop. The requirement for fault tolerant computation is fulfilled, as $j$ and $k$ can be any pair of qubits.

The second example is QC using symmetric SQUID loop $\left(E_{J m}=E_{J 0}\right)$. The Hamiltonian is given by $H=H_{0}+H_{1}$ $+\mathrm{H}_{2}$, where

$$
\begin{gathered}
H_{1}=-\frac{1}{2} \sum_{j}^{N} E_{J}^{0}\left(\phi_{j}\right)\left(e^{-i g\left(a+a^{\dagger}\right)} \sigma_{j}^{+}+\text {H.c. }\right), \\
H_{2}=E_{c} \sum_{\langle i, j\rangle}\left(\bar{n}_{i}-n_{i}\right)\left(\bar{n}_{j}-n_{j}\right),
\end{gathered}
$$

with $E_{J}^{0}\left(\phi_{j}\right)=2 E_{J 0} \cos \left(\pi \phi / \phi_{0}\right)$. Here $H_{0}$ is the same as that of Eq. (6), and $\mathrm{H}_{2}$ with $\langle i, j\rangle$ denoting the nearest-neighbor qubits represents the capacitive couplings between qubits [5]. We also consider this coupling because it is unlikely to work out easily a nontrivial two-qubit gate by using only the coupling with cavity.

The single-qubit gates may be realized when $\mathrm{H}_{2}$ is set to zero. In the rotationed frame $U_{0}(t)=\exp \left[-i \nu t\left(a^{\dagger} a\right.\right.$ $+1 / 2)] \exp \left(-i E_{n_{k}} t \sigma_{j}^{z}\right)$, the interaction Hamiltonian is given by $H_{i n t}^{\prime}=U_{0}^{\dagger} H_{\text {int }} U_{0} \approx H_{a}+H_{b}$, where

$$
\begin{gathered}
H_{a}=-\sum_{j}^{N} E_{J}^{0}\left(\phi_{j}\right) \sigma_{j}^{x}\left(E_{n_{k}}^{-}=0\right), \\
H_{b}=\frac{1}{2} \sum_{j}^{N} E_{J}^{0}\left(\phi_{j}\right)\left(i g a \sigma_{j}^{+}+\text {H.c. }\right)\left(E_{n_{k}}^{-}=\nu\right) .
\end{gathered}
$$

Thus from Eq. (13) we have a unitary operator $U_{x}\left(\gamma_{k}^{x}\right)$ $=\exp \left(-i \gamma_{k}^{x} \sigma_{k}^{x} / 2\right)$, with $\gamma_{k}^{x}=2 E_{J}^{0}\left(\phi_{k}\right) t / \hbar$ for the qubit $k$ by choosing $E_{n_{k}}=0$. On the other hand, it is seen from Eq. (14) that the interaction between the cavity and qubit $k$ is decoupled by choosing $\phi_{k}=\left(i_{k}+1 / 2\right) \phi_{0}$, with $i_{k}$ an integer, and the evolution operator is derived as $U_{z}\left(\gamma_{k}^{z}\right)=\exp \left(-i \gamma_{k}^{z} \sigma_{k}^{z} / 2\right)$ with $\gamma_{k}^{z}=2 E_{n_{k}} t / \hbar$. The gates described by $U_{x}\left(\gamma_{k}^{x}\right)$ and $U_{z}\left(\gamma_{k}^{z}\right)$ are a well-known universal set of single-qubit gates.

Also by choosing $\phi_{k}=\left(i_{k}+1 / 2\right) \phi_{0}$, the Hamiltonian of two qubits becomes $H=E_{n_{1}}^{-} \sigma_{1}^{z}+E_{n_{2}}^{-} \sigma_{2}^{z}+E_{c}\left(\bar{n}_{1}-n_{1}\right)\left(\bar{n}_{2}\right.$ $\left.-n_{2}\right)$. Then we find a conditional phase gate in computational basis given by

$$
U=\operatorname{diag}\left(e^{i \gamma_{00}}, e^{i \gamma_{01}}, e^{i \gamma_{10}}, e^{i \gamma_{11}}\right),
$$

where $\gamma_{n_{1} n_{2}}=-\omega_{n_{1} n_{2}} t$ with $\hbar \omega_{n_{1} n_{2}}$ the eigenenergy of state $\left|n_{1} n_{2}\right\rangle$, it is nontrivial under the condition $\gamma_{00}+\gamma_{11} \neq \gamma_{01}$ $+\gamma_{10}(\bmod 2 \pi)$. A similar gate was addressed in Refs. $[5,6]$. The gates $U_{x}\left(\gamma_{k}^{x}\right), U_{z}\left(\gamma_{k}^{z}\right)$ and Eq. (15) consist of a universal set of quantum gates using charge qubits in symmetric SQUID loop.

It is remarkable that the previously proposed geometric quantum gates $[5-7,16]$ are still workable in the above two examples, as the Hamiltonians are essentially in the same forms. Thus an intrinsically fault tolerant QC is possible in the present systems. On the other hand, the scheme based on symmetric SQUID loop has some special advantages compared with that using asymmetric SQUID. First, the coupling between the cavity and charge qubits may be experimentally tunable to zero (but cannot touch zero for asymmetric case). Thus the two-qubit gates may be accomplished by using the same approach as that in Refs. [5,6]. Second, the eigenstates of the Hamiltonian may be tuned to degenerate. Then the relative phase of two logical states is zero during idle periods, but the nondegenerate feature in asymmetric SQUID loop requires that the phase difference induced by the energy spacing between logical states must be controlled with high accuracy [3].

Another main advantage for coupling by cavities is on the quantum communication. Since swapping gates are essential in this direction, we now address three very useful kinds of restricted swapping gates based on the cavity QED technique in the symmetric SQUID. Note that a slight modification of the approach can also be applicable in the nonsymmetric SQUID.

If we consider a fixed qubit $k$ and pursue the evolution of the system followed by Eq. (14) for a certain time $t$, we obtain an evolution operator

$$
U_{k p}\left(\Gamma_{k} t\right)=\exp \left[-i \Gamma_{k} t\left(i \sigma_{k}^{\dagger} a+\text { H.c. }\right)\right],
$$

with $\Gamma_{k}=g E_{J}^{0}\left(\phi_{k}\right) / 2 \hbar$. This transformation keeps the state $\left|0_{k}\right\rangle|0\rangle_{p h}$ unaltered, whereas

$$
\begin{aligned}
& \left|0_{k}\right\rangle|1\rangle_{p h} \rightarrow \cos \left(\Gamma_{k} t\right)\left|0_{k}\right\rangle|1\rangle_{p h}+\sin \left(\Gamma_{k} t\right)\left|1_{k}\right\rangle|0\rangle_{p h}, \\
& \left|1_{k}\right\rangle|0\rangle_{p h} \rightarrow \cos \left(\Gamma_{k} t\right)\left|1_{k}\right\rangle|0\rangle_{p h}-\sin \left(\Gamma_{k} t\right)\left|0_{k}\right\rangle|1\rangle_{p h},
\end{aligned}
$$

with the subscript " $k$ " (" $p h$ ") denoting the $k$ th qubit (photon in the cavity). Then we have the following transformation:

$$
\left(\alpha\left|0_{k}\right\rangle+\beta\left|1_{k}\right\rangle\right)|0\rangle_{p h} \stackrel{U_{k p}^{\prime}}{\longrightarrow}\left|0_{k}\right\rangle\left(\alpha|0\rangle_{p h}+\beta|1\rangle_{p h}\right)
$$


between qubit $k$ and photon in the cavity, where $\alpha$ and $\beta$ are complex numbers. Here $U_{k p}^{\prime}$ is a short denotation of $U_{k p}[(2 n-1 / 2) \pi]$, with $n$ an integer. Gate (17) is the basis for interconverting stationary and flying qubits. Moreover, we find that a swapping gate between qubits $k$ and $j$ in the same cavity is given by the operator $U_{j p}^{\prime} U_{k p}^{\prime}$, i.e.,

$$
\left(\alpha\left|0_{k}\right\rangle+\beta\left|1_{k}\right\rangle\right)\left|0_{j}\right\rangle|0\rangle_{p h} \stackrel{U_{j p}^{\prime} U_{k p}^{\prime}}{\longrightarrow}\left|0_{k}\right\rangle\left(\alpha\left|0_{j}\right\rangle+\beta\left|1_{j}\right\rangle\right)|0\rangle_{p h} .
$$

It is worth pointing out that $j$ and $k$ may be any two qubits in the same cavity, then this gate can help to realize any twoqubit gate acting on any pair of qubits as long as it can be achieved on two fixed qubits. Thus this swapping gate is very useful to overcome the drawback arising from that the two-qubit gates described by Eq. (15) act only on the nearest neighbor qubits.

Moreover, the ideal quantum transmission [12] (swapping gate) between two cavities 1 and 2 [see Fig. 1(c)]

$$
\begin{aligned}
& \left(\alpha\left|0_{1}\right\rangle+\beta\left|1_{1}\right\rangle\right)\left|0_{2}\right\rangle \otimes\left|0_{1}\right\rangle_{p h}\left|0_{2}\right\rangle_{p h}|\mathrm{vac}\rangle \\
& \quad \rightarrow\left|0_{1}\right\rangle\left(\alpha\left|0_{2}\right\rangle+\beta\left|1_{2}\right\rangle\right) \otimes\left|0_{1}\right\rangle_{p h}\left|0_{2}\right\rangle_{p h}|\mathrm{vac}\rangle,
\end{aligned}
$$

with $|v a c\rangle$ the vacuum state of the free electromagnetic modes connecting the cavities, can be accomplished by appropriately selecting the controllable parameters $\bar{n}_{i}$ and $\phi_{i}$ in each cavity. Following the approach described in Ref. [12], we find that the evolution equations to achieve the ideal transmission in Eq. (18) are given by

$$
\dot{\alpha}_{i}=g \beta_{i} E_{J}^{i} / 2, \quad \dot{\beta}_{i}=-g \alpha_{i} E_{J}^{i} / 2-\kappa \beta_{1} \quad(i=1,2),
$$

where $\kappa$ is the loss rate of each cavity, $\left\{\alpha_{1}, \alpha_{2}, \beta_{1}, \beta_{2}\right\}$ are the expansion coefficients of the wave function $|\Psi(t)\rangle$ in the basis $\left\{\left|1_{1} 0_{2}\right\rangle\left|0_{1} 0_{2}\right\rangle_{p h},\left|0_{1} 1_{2}\right\rangle\left|0_{1} 0_{2}\right\rangle_{p h},\left|0_{1} 0_{2}\right\rangle\left|1_{1} 0_{2}\right\rangle_{p h}\right.$, $\left.\left|0_{1} 0_{2}\right\rangle\left|0_{1} 1_{2}\right\rangle_{p h}\right\}$, and we have chosen $\bar{n}_{i}$ so that $E_{n_{i}}^{-}=\nu / 2$.
The mathematical problem is now to find $\phi_{i}(t)$ such that $\alpha_{1}(-\infty)=\alpha_{2}(+\infty)=1$ and Eq. (19) are fulfilled. A type of symmetric solutions $\left[E_{J}^{0}\left(\phi_{2}(t)\right)=E_{J}^{0}\left(\phi_{1}(-t)\right)\right]$ can be found by the approach outlined in Ref. [12]. For example, we find that

$$
\begin{gathered}
\phi_{1}=\left(\phi_{0} / \pi\right) \arccos \left(\kappa / g E_{J 0}\right), \\
\phi_{2}=\left(\phi_{0} / \pi\right) \arccos \left[\kappa e^{-\kappa t / 2} \cos (\sqrt{3} \kappa t / 2-\pi / 3) / g \alpha_{2} E_{J 0}\right],
\end{gathered}
$$

where $\alpha_{2}=\sqrt{1-e^{-\kappa t}[1+\cos (\sqrt{3} \kappa t-\pi / 6) / \sqrt{3}] / 2}(t \geqslant 0)$, is a set of appropriate analytical solutions. Therefore the ideal quantum transmission between two nodes of a quantum network may be accomplished using microwave photons in this system [12].

The quantum network proposed here consists of spatially separated nodes connected by quantum communication channels. Each node is a quantum computer using Josephson junctions, which is able to store quantum information in quantum bits and processes this information locally using quantum gates. The transmission between the nodes of the network is accomplished using microwave photons via the cavity QED technique [17].

To conclude, we have presented a different approach to coupling Josephson-junction qubits and have shown that this system satisfies all the acknowledged theoretical criteria for the construction of quantum-information network. Nevertheless, it is a big challenge to implement this kind of network experimentally.

This work was supported by the RGC grant of Hong Kong under Grant No. HKU7114/02P and a URC fund of HKU. S.L.Z was supported in part by SRF for ROCS, SEM, the NSF of Guangdong under Grant No. 021088, and the NNSF of China under Grant No. 10204008.
[1] A. Shnirman, G. Schön, and Z. Hermon, Phys. Rev. Lett. 79, 2371 (1997).

[2] D.V. Averin, Solid State Commun. 105, 659 (1998).

[3] Y. Makhlin, G. Schön, and A. Shnirman, Nature (London) 398, 305 (1999); Rev. Mod. Phys. 73, 357 (2001).

[4] Y. Nakamura, Yu.A. Pashkin, and J.S. Tsai, Nature (London) 398, 786 (1999).

[5] G. Falci, R. Fazio, G.M. Palma, J. Siewert, and V. Vedral, Nature (London) 407, 355 (2000).

[6] S.L. Zhu and Z.D. Wang, Phys. Rev. Lett. 89, 097902 (2002); 89, 289901(E) (2002); Phys. Rev. A 66, 042322 (2002).

[7] X.B. Wang and M. Keiji, Phys. Rev. B 65, 172508 (2002).

[8] Yu.A. Pashkin, T. Yamamoto, O. Astafiev, Y. Nakamura, D.V.
Averin, and J.S. Tsai, Nature (London) 421, 823 (2003).

[9] D.P. DiVincenzo, Fortschr. Phys. 48, 771 (2000).

[10] J. Preskill, Proc. R. Soc. London, Ser. A 454, 385 (1998).

[11] P. Barbara, A.B. Cawthorne, S.V. Shitov, and C.J. Lobb, Phys. Rev. Lett. 82, 1963 (1999).

[12] J.I. Cirac, P. Zoller, H.J. Kimble, and H. Mabuchi, Phys. Rev. Lett. 78, 3221 (1997).

[13] W.A. Al-Saidi and D. Stroud, Phys. Rev. B 65, 224512 (2002).

[14] S. Lloyd, Phys. Rev. Lett. 75, 346 (1995).

[15] A.M. Childs and I.L. Chuang, Phys. Rev. A 63, 012306 (2000).

[16] S.L. Zhu and Z.D. Wang, Phys. Rev. A 67, 022319 (2003).

[17] G.P. He, S.L. Zhu, Z.D. Wang, and H.Z. Li, Phys. Rev. A 68, 012315 (2003). 